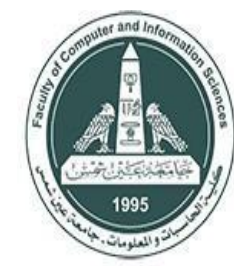

International Journal of Intelligent Computing and Information Sciences

https://ijicis.journals.ekb.eg/

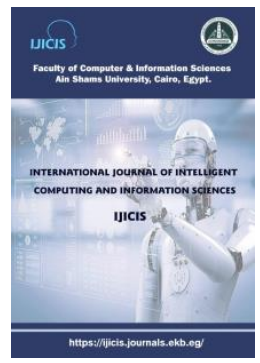

\title{
MACHINE AND DEEP LEARNING APPROACHES FOR HUMAN ACTIVITY RECOGNITION
}

\author{
Maha Mohammed Alhumyyani*, \\ Department of Information \\ Systems, Faculty of Information \\ and Computer Science, Ain Shams \\ University,Cairo, Egypt \\ mwiexs@gmail.com
}

\author{
Rasha Ismail, \\ information Systems Department \\ Faculty of Computer and \\ Information Science, Ainshams \\ University, Egypt , \\ rashaismail@yahoo.com
}

\author{
Mahmoud Mounir, \\ information Systems Department \\ Faculty of Computer and \\ Information Science, Ainshams \\ University, Egypt \\ Mahmoud.mounir@cis.asu.edu.eg
}

Received 2021-6-23; Revised 2021-8-31; Accepted 2021-8-31

\begin{abstract}
Human Activity Recognition (HAR) is a domain that has shown great interest in the past years and tills now. The main cause for this is that it can be used in various applications. There exist several devices and sensors that can capture and record activities. In this paper, a survey about the machine learning and deep learning methodologies in HAR is provided with information about the data, filtering methods, feature extraction methods, classification, and different performance measurements. The main aim is to present the machine learning and deep learning methodologies used in HAR. Therefore, the methods that showed the highest performance can be presented and investigated. In addition to this, the survey will cover the types of actions or activities that are predicted. Then, the results obtained from the survey are discussed to explore the most efficient methods in both machine and deep learning for the recognition of HAR. Moreover, the results involves illustrating whether the deep or machine learning methods is better in terms of data size, enhance performance, and number of activities to be recognized. Finally, the conclusions, limitations, and challenges in HAR are presented clearly.
\end{abstract}

\section{Keywords}

Human activity recognition, Machine learning, Deep learning, smartphone, sensors

\section{Introduction}

Human activity recognition (HAR) can be known with various types of definitions. HAR is preserved to be a field of studying and identifying the movements of the individuals or the action of the human based on sensor data [1]. These movements can be different activities such as walking, talking, standing, and sitting. They are also called indoor activities. On one hand, the data can be remotely recorded based on some specific devices such as radars, videos, or any other types of wireless methodologies. On the other hand, the data can be recorded directly from subjects that carry some hardware devices such as smartphones. These devices have sensors such as accelerometers and gyroscopes. The sensor can capture data efficiently. HAR is related to the automatic detection of the different physical activities that are adapted by people during their daily lives [2].

Any HAR can assist in identifying the activities done by a specific individual and it also can provide informative feedback for interference. Activities can be categorized into three classes as described in figure.1. The first class is

* Corresponding author: Maha Mohammed Alhumyyani

Department of Information Systems, Faculty of Information and Computer Science, Ain Shams University,Cairo, Egypt

E-mail address: mwiexs@gmail.com 
known as the ambulation activities and they are defined as waling up and down stairs, jogging and they are down day by day [3]. The second class is the activity that is based on fitness and these activities are performed by the young people and the adults that are less than 30 years old. These activities give them that chance to perform the fitness day by day. The third and the last class is known as the activities that can be functional. These activities are like sweeping, cleaning the trash, taking different phone calls, cooking food, washing and brushing hands and teeth, wearing shoes and jacket, combing the hair and adding perfume [4].

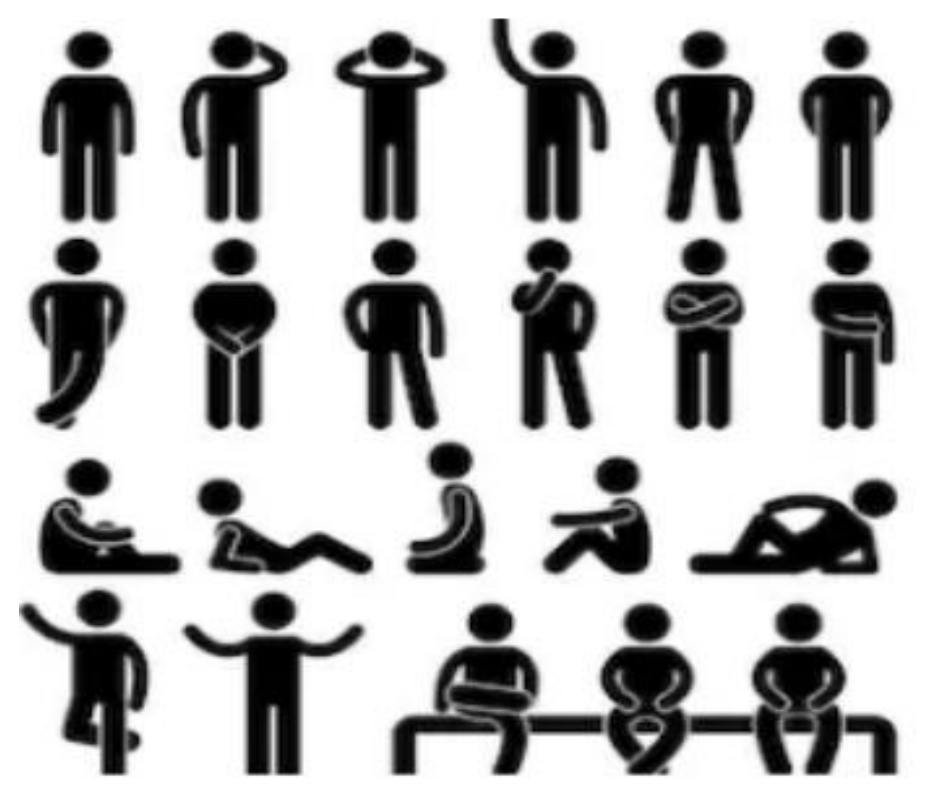

Figure 1 Examples of action-based activities [4]

There exist different types of sensors that can be used to capture different types of activities and movements. These devices can be videos, motion sensors, cameras, radars [5], acoustic sensors [6], echo [7], food scale [8], device-free sensing. These devices can assist in detecting the actions and activities from the individuals. Some of these systems face a lot of problems and challenges that are more related to privacy and space restrictions. Therefore, different types of sensors were introduced based on accelerometers and gyroscopes. These sensors are sometimes known as wearable sensors. The former sensors can overcome privacy and security issues [9].

HAR has gained a lot of attention in the past recent years. It also has been used in many different domains and applications. These applications can be mobile computing, context-aware computing, surveillance, and many others. Therefore, a survey about the methodologies and techniques used in the HAR is provided in detail to determine the progress made in HAR. The remainder of the paper is organized as follows: Section 2 represents the related works on machine learning in HAR. Section 3 specifies the deep learning methodologies in HAR. Section 4 describes the discussion. Finally, section 5 represents the conclusion and future works.

\section{Machine learning (ML) in HAR}

One of the most important domains and considered more challenging for the researchers is the HAR. This is because it involves the recognition of complex movements or actions and also the number of unusual activities that may exist. Therefore, some researchers begin on working on HAR and representing it as a traditional pattern classification problem. The main common machine learning methods used in HAR is the Hidden Markov, support vector machine (SVM). Then, after that a great shift has been done from the big data methods and machine learning approaches to deep learning methods. Moreover, a type of learning is known as shallow learning that is based on that is based on feature engineering from the inputs that are obtained from sensors. The data can be a sort of heuristic-driven or based on the knowledge taken from the human. The model developed for a specific domain can be extended to another one. So shallow learning is more convenient for recognize activities that has low level. Also, we cannot capture any sort of activities that are considered complex especially the ones that must require a sequence of different micro activities using shallow learning methods. 
Some of the pioneer approaches were applied in the HAR using an accelerometer, and it was published way back in the 90s [10]. The studies that were able to show or produce efficient results that are satisfactory depended on multiple or different sensors that are added on several or different parts of the body fused with machine learning methods [11]. One study proposed by kwapisze et al, [12] used a hand-crafted system for obtaining features from the sensor data. They applied different types of machine learning methods for classification. Several classifiers were applied such as J48, logistic regression, multi-layer perceptron (MLP), and Strawman. The accuracies resulted by J48, logistic regression, MLP, and strawman are 85.1\%, 78.1\%, 91.7\%, and 37.2\% respectively. MLP and J48 decision trees have shown the highest performance in terms of accuracy compared with other machine learning methods. The maximum accuracy reached was by MLP with a value of $91.7 \%$ as mentioned previously.

Another study was proposed by Sharma et al. [13] depending on the neural network for the recognition of human movements based on a tri-axial accelerometer sensor. A 4-layer backpropagation network with levenbergmarqudrat used for training showed the highest performance. Three different activities were used in this study for recognition which are based on rest, walk, and run. The data of these activities were captured from the tri-axial accelerometer. The results obtained from the network proposed in this study was $83.96 \%$. Khan et al. [14] applied a machine learning methodology using a decision tree classifier. The data was obtained or taken based on a Wii remote which is considered to be a readymade, lightweight, small, and easy device to recognize basic physical activities. Shoaib et al. [15] proposed a methodology to classify five types of activities. Four different feature sets were extracted in both time and frequency domains. These features are based on the mean, standard deviation, median, zero crossings, root mean square, and many others. In addition to this, seven classifiers were used and applied to these data, and random forest (RF) showed the highest performance. Anguita et al. [16] proposed a system that uses smartphone sensors to recognize the HAR. The methodology proposed is based on a regular SVM classifier and it also depends on fixed-point arithmetic to decrease the computational costs. The accuracy obtained from the model was $89 \%$.

Another study presented by Tran et al. [17] for the recognition of six main actions which are based on walking, standing, sitting, lying down, up, and downstairs. The activities were captured by sensors from the mobile. A set of features were selected such as the difference in the sensors data, magnitude, fast Fourier transform, length of the window, mean, standard deviation, maximum, minimum, energy and entropy. The SVM classifier was used and the quality of the features and the quality of the model training contribute to enhancing the performance. The number of features obtained from this model is 248 . The accuracy reached from the proposed system was $89.59 \%$.

Gusain et al. [18] implemented a methodology based on an extreme gradient boosted decision trees eXtreme (GBM). The data set used was HAPT and the number of instances was 10,929 and the number of attributes used was 561. Several classifiers were applied and the XGBoost showed the highest accuracy performance and it was $94.9 \%$.

Zdravevski et al. [19] have proposed a nonexclusive include building approach to choose vigorous characteristics from a assortment of sensors that can be utilized to create precise classification models. A few time and recurrence space highlights have been extricated within the at first enrolled time arrangement and a few recently made time arrangement such as quick Fourier change arrangement, to begin with subsidiaries, extents, and Delta arrangement. Moreover, the number of capacities produced is significantly decreased with a twophase work choice. At last, different classification models are prepared and tried in a partitioned test collection. Six different classifiers were used such as K-nearest neighbor (KNN), linear regression (LR), naïve bias (NB), random forest (RF), extremely randomized trees (ERT), and SVM.

Bao et al. [20] proposed a methodology based on machine learning for the recognition of human activities. The data were collected from 20 subjects, and the feature was extracted based on the frequency-domain entropy, and correlation of acceleration data. Several classifiers were applied and the decision trees (DT) are the ones that showed the highest performance with an overall accuracy of 84\%. In addition to this, Shoaib et al. [21] proposed another study based on a methodology for the identification of the HAR based on a filtering method using a simple difference filter. The features were extracted from the data based on the mean, standard deviation. The classification was based on the decision tree achieving an accuracy of 93.91\%. Khan et al. proposed [22] an approach for recognizing different human activities based on data obtained from a triaxle accelerometer. The 
features were extracted by the linear discernment analysis, and the classification is done based on ANN. The system was able to recognize 15 activities with an accuracy of $97.9 \%$.

\section{Deep learning (DL) in HAR}

One of the recent and new methods is the deep learning as it can extract features from the input and this can prevent the problem of the features that are obtained based on hand-crafted. In addition to this, the famous deep learning approaches starts with convolutional neural network $(\mathrm{CNN})$ as it considered to be very successful in many different application based on learning complex activities and actions. The property of CNN is that it does not depend on the invariance in the scale. Moreover, HAR suffer from challenges that can be attached with the ground truth annotation, identification of a specific activity in case of different various users. In addition to this, several other factors such as sensors with fault values, sensing devices heterogeneity, activity model deployment in the form of one type of domain to another. The activity model must be feed with a huge number of labelled data so that supervised machine learning tools can be applied to learn the hidden patterns in the phase of the training. Deep learning works by learning the patterns or the hidden pattern between the inputs during the training stage. The stage of labels the ground truth for the data collected from the sensor is not even feasible. One of the solutions to tackle these problems is the application of active learning. In active learning, the model can actively search the user for labels. In addition to this, processing a large number of datasets can cause high computational costs and increase the training time of the model. Therefore, the researcher started to investigate the transfer learning approach as this approach permits the transfer of the knowledge obtained from one model to another, this will permit that new models to do training with a small amount of training features leading to a reduction in the computational cost.

Cheng et al. [23] developed a deep neural model based on the combination between the inception neural network and the recurrent neural network. Three different datasets were used and they are the opportunity, PAMAP2, and smartphones dataset. The resulted obtained from the inception neural network only are $85.1 \%, 88 \%, 91.5 \%$ respectively on the three datasets, while the RNN model showed accuracies of $91.5 \%, 90.5 \%$, and $91 \%$ on the same three datasets respectively. The accuracy resulted on the three different datasets using the proposed methodology was $94.6 \%, 93.5 \%$, and 94.5\%. Hossain et al. et al. [24] applied different methodologies for the recognition of the HAR. The methods are based on convolutional neural network (CNN), long short term memory (LSTM), Bidirectional long short term memory (BiLSTM), multilayer perception (MLP), and support vector machine (SVM). Two main datasets were utilized for experimentation which are UCI and Pamap2 datasets. The UCI dataset has 6 main activities, while the Pamap2 dataset has 12 activities and the accuracy performance of these datasets reached its maximum using CNN with an average accuracy of $93.21 \%$ for the UCI dataset and $91.0 \%$ for the Pamap2 dataset. Another methodology is based on deep learning for the recognition of different activities [25]. These activities are still, walk, run, bike, car, bus, train, subway based on smartphone sensor data. A deep multimodal spectro-temporal Resnet model is applied to train the data. Another model based on the hidden Markov model was presented and also a baseline DL model was illustrated. Finally, all these models are fused. The classification was based on Metamodels such as RF, Boosting, Bagging, SVM, KNN, DT, and Bayes. The results are $94.9 \%$ accuracy based on the fused model with SVM.

Zebian et al. [26] proposed a deep learning model based on CNN for the classification of different human activities. The inputs are multichannel time-series signals that are obtained from a set of body-worn sensors. The CNN model was based on a set of convolutional layers and different hyper-parameters. The performance was compared with SVM and MLP. Deep CNN showed the highest performance with an accuracy of $97.01 \%$. Another deep learning approach is proposed based on a deep convolutional generative adversarial network by Shi et al. [27]. In addition to this, a transfer learning deep CNN (DCNN) for feature extraction and classification was proposed. The pertained model is the imagenet, and the results showed that the combination of the DCNN and the pertained model showed the highest performance. Another deep learning model was proposed by Cruz [28] et al. based on three parallel CNNs used for local feature extraction. The three CNN models are known as fine-CNN, medium-CNN, and coarseCNN. These models are fused in the classification stage. The resulted parallel model approached an accuracy that is approximate to $100 \%$ for classifying six main activities. 
Chen et al. [29] applied a method for the recognition of different activities based on CNN. The convolutional kernel in the proposed $\mathrm{CNN}$ is adjusted to adapt the features of the tri-axial acceleration signals. Eight main different activities were used with 31688 samples. The experiments showed an accuracy of $93.8 \%$ without any need for feature extraction methodologies. Another approaches proposed by Mukherjee et al. [30] for the recognition of human activities are the CNN-Net, Encoded-Net, and CNN-LSTM which is defined by EnsemConvNet. The methods were applied on different datasets such and WISDM and MobiAct. The results showed that EnsemConvent combined with an adaptive weight fusion approach (AWFA) proved the highest accuracy with 97.1\%. Another method is applied by Almasluksh et al. [31] to produce a reliable smartphone-based HAR. A deep CNN model is proposed to provide a robust position-independent HAR system. The dataset selected is based on a real-world HAR public dataset. The performance of the proposed method was compared with other machine learning methods. The result proved a great significance ranging from $84 \%$ to $88 \%$ for position-independent HAR and 89 to $98 \%$ for position detection.

Masum et al. [32] proposed a deep learning model for the recognition of the HAR. This model was compared with four machine learning classifiers which are RF, NB, SVM, and MLP. The MHEALTH dataset was used to determine the HAR process and twelve activities were used during the classification stage. The deep CNN showed the highest accuracy performance with an accuracy of $98.4 \%$. Finally, Ihianle et al. [33] proposed a method based on deep learning methods for the identification of HAR. The proposed methods are based on CNN and Bi-LSTM. The Bi-LSTM proved its advantage because it moves forward and backward in a defined sequence to improve the extracted features. A final model was proposed based on multi-channel convolutional bidirectional LSTM (MCBLSTM). Two main datasets were used to determine the performance of the proposed methods which as the MHEALTH and WISDM. The accuracy proposed from MCBLSTM showed the highest performance using both datasets with a value of $97.7 \%$.

\section{Discussion}

The review shows techniques applied in HAR based on machine learning and deep learning. Each study presented shows a set of phases to be performed to recognize a set of actions. These phases are data acquisition, feature extraction, and classification. Table. 1 and 2 show the details of each related work in terms of authors, year of publication, activities or actions to be recognized, methodology, and the final classification accuracy. In the data acquisition phase, it can be revealed that the most commonly used data sets in HAR are the UCI, Pampap2, smartphones datasets. A few studies applied or gathered data by themselves. In the feature extraction phases, the studies manifested several machine learning and deep learning methods. The machine learning methods revealed in the HAR studies for feature extraction are mean, the standard deviation of the data, frequency, and correlation of the data obtained from sensors, and linear discernment analysis.

The deep learning method applied in extracting features in HAR is based on CNN, LSTM, Bi-LSTM, deep CNN, DCNN, Fine-CNN, Medium-CNN, Coarse-CNN, Encoded-Net, EnsemConvNet with AWFA, and MCBiLSTM. Then, in the classification stage, several classifiers were applied for the recognition and identification of the activities. These classifiers are ANN, MLP, NB, SVM, DT, GBM, XGBoost, and RF. The studies illustrated a huge number of activities to be recognized ranging from 5 to 25 activities. These activities can be walking, standing, sitting, laying down, upstairs, downstairs, biking, running, jumping, climbing, cooking, performing office work, relaxing, jogging, knees bending, cycling, and many other activities. These actions can contribute in many different applications and domains. Finally, on one hand it can be seen that the deep learning methods achieve a higher performance accuracy than the machine learning methods. Based on the studies used in the survey it can be manifested that the highest accuracy achieved based on machine learning method is $98.9 \%$ on four activities and when the number of activities started to increase the performance begins to degrade consequently. On the other hand, the highest performance accuracy reached using deep learning methods is $100 \%$ using six activities. The rest of the deep learning approaches based on the survey obtain higher performance than the machine learning methods. 
Table. 1 Machine Learning Methodologies used in HAR

\begin{tabular}{|c|c|c|c|c|}
\hline Authors & Year & Number of Actions & Methodology & Accuracy \\
\hline $\begin{array}{l}\text { kwapisze } \\
\text { et al, [12] }\end{array}$ & 2011 & $\begin{array}{c}\text { Walking, Jogging, Upstairs, } \\
\text { Downstairs, Sitting, and } \\
\text { Standing }\end{array}$ & $\begin{array}{l}\text { Handcrafted features } \\
\text { based on average and } \\
\text { standard deviation of } \\
\text { the data }+\mathrm{J} 48, \\
\text { logistic regression, } \\
\text { MLP, and StawMan }\end{array}$ & $\begin{array}{c}\mathrm{J} 48 \text { highest } \\
\text { performance with } \\
\mathrm{A}=91.7 \%\end{array}$ \\
\hline $\begin{array}{l}\text { Sharma et } \\
\text { al., } \\
\text { [13] }\end{array}$ & 2008 & Rest, Walk, and Run & $\begin{array}{l}\text { Neural network with } \\
\text { levenberg } \\
\text { marquadrat }\end{array}$ & $\begin{array}{c}\text { Without ANN } \\
\text { A }=75.0 \% \\
\text { With ANN } \\
\text { A }=83.9 \%\end{array}$ \\
\hline $\begin{array}{l}\text { Khan et al. } \\
\text { [14] }\end{array}$ & 2012 & $\begin{array}{c}\text { Lying, Sitting, Walking, and } \\
\text { Running }\end{array}$ & Decision trees & $\mathrm{A}=98.9 \%$ \\
\hline $\begin{array}{l}\text { Shoaib et } \\
\text { al. [15] }\end{array}$ & 2014 & $\begin{array}{l}\text { Standing, sitting, biking, } \\
\text { walking up and downstairs }\end{array}$ & $\begin{array}{c}\text { Four different } \\
\text { features sets }+ \text { seven } \\
\text { types of classifiers }\end{array}$ & $\begin{array}{c}\text { RF showed the } \\
\text { highest accuracy } \\
\mathrm{A}=94 \%\end{array}$ \\
\hline $\begin{array}{l}\text { Anguita et } \\
\text { al. [16] }\end{array}$ & 2012 & $\begin{array}{c}\text { Walking, Standing, Sitting, } \\
\text { Lying down, Up and } \\
\text { Downstairs }\end{array}$ & Adaptive SVM & $89.3 \%$ \\
\hline $\begin{array}{c}\text { Tran et al. } \\
\text { [17] }\end{array}$ & 2016 & $\begin{array}{c}\text { Walking, Standing, Sitting, } \\
\text { Lying down, Up and } \\
\text { Downstairs }\end{array}$ & $\begin{array}{c}248 \text { features of high } \\
\text { quality + SVM }\end{array}$ & $89.59 \%$ \\
\hline $\begin{array}{l}\text { Gusain et } \\
\text { al. [18] }\end{array}$ & 2018 & $\begin{array}{l}\text { Standing and lying, } \\
\text { Standing and Sitting }\end{array}$ & $\begin{array}{c}\text { Extreme gradient } \\
\text { boosted decision tree } \\
\text { eXtreme }(\mathrm{GBM})+ \\
\text { XGBoost }\end{array}$ & $\mathrm{A}=94.97 \%$ \\
\hline $\begin{array}{l}\text { Zdravevski } \\
\text { et al. [19] }\end{array}$ & 2017 & $\begin{array}{l}25 \text { types of actions or } \\
\text { different activities }\end{array}$ & $\begin{array}{c}\text { Data were extracted } \\
\text { based on the original } \\
\text { and magnitude time } \\
\text { series }+6 \text { main } \\
\text { classifier }\end{array}$ & $\begin{array}{l}\text { SVM has shown } \\
\text { the highest } \\
\text { accuracy } \\
\mathrm{A}=96.0 \%\end{array}$ \\
\hline $\begin{array}{l}\text { Bao et al. } \\
\quad[20]\end{array}$ & 2004 & $\begin{array}{l}20 \text { different types of } \\
\text { ambulation activities }\end{array}$ & $\begin{array}{c}\text { Frequency and } \\
\text { correlation features }+ \\
\text { Decision trees }\end{array}$ & $\mathrm{A}=84 \%$ \\
\hline $\begin{array}{l}\text { Shoaib et } \\
\text { al. [21] }\end{array}$ & 2009 & $\begin{array}{l}\text { Walking, Running, Standing, } \\
\text { Ascending, and Descending }\end{array}$ & $\begin{array}{c}\text { The mean and } \\
\text { standard deviation of } \\
\text { data }+ \text { decision tree } \\
\text { with pruning }\end{array}$ & $A=93.91 \%$ \\
\hline $\begin{array}{l}\text { Khan et al. } \\
\text { [22] }\end{array}$ & 2010 & 15 different activities & $\begin{array}{l}\text { Linear discernment } \\
\text { analysis + ANN }\end{array}$ & $\mathrm{A}=97.9 \%$ \\
\hline
\end{tabular}


Table. 2 Deep learning Methodologies used in HAR

\begin{tabular}{|c|c|c|c|c|}
\hline Authors & Year & Number of Actions & Methodology & Accuracy \\
\hline $\begin{array}{l}\text { Cheng et } \\
\text { al. [23] }\end{array}$ & 2017 & $\begin{array}{l}\text { Walking, Standing, Sitting, } \\
\text { Lying down, Up and } \\
\text { downstairs }\end{array}$ & (InnoHAR) & $94.5 \%$ \\
\hline $\begin{array}{l}\text { Hossain et } \\
\text { al. [24] }\end{array}$ & 2019 & $\begin{array}{l}\text { UCI: Walking, Standing, } \\
\text { Sitting, Lying down, Up and } \\
\text { Downstairs } \\
\quad \text { Pamap2: } 12 \text { actions }\end{array}$ & $\begin{array}{c}\text { CNN } \\
\text { LSTM } \\
\text { BiLSTM } \\
\text { MLP } \\
\text { SVM }\end{array}$ & $\begin{array}{l}\text { CNN showed the } \\
\text { highest accuracy } \\
\text { performance of } \\
93.21 \% \text { for UCI } \\
91.0 \% \text { for Pamap2 }\end{array}$ \\
\hline $\begin{array}{l}\text { Gjoreski et } \\
\text { al. [25] }\end{array}$ & 2020 & $\begin{array}{l}\text { Still, walk, bike, car, bus, } \\
\text { train, subway }\end{array}$ & SVM & F1-Score $=94.9 \%$ \\
\hline $\begin{array}{l}\text { Zebin et } \\
\text { al. [26] }\end{array}$ & 2016 & $\begin{array}{l}\text { Walk, walking upstairs, } \\
\text { downstairs, sitting, standing, } \\
\text { laying down }\end{array}$ & $\begin{array}{c}\text { Deep CNN } \\
\text { SVM } \\
\text { MLP } \\
\end{array}$ & $\begin{array}{l}A=97.01 \% \\
A=96.4 \% \\
A=91.7 \%\end{array}$ \\
\hline $\begin{array}{l}\text { Shi et al. } \\
\text { [27] }\end{array}$ & 2018 & $\begin{array}{l}\text { Slow walking, Fast walking, } \\
\text { Really fast walking }\end{array}$ & $\begin{array}{c}\text { DCNN } \\
\text { Pertained Model + } \\
\text { DCNN }\end{array}$ & $\begin{array}{l}A=99.82 \% \\
A=99.91 \%\end{array}$ \\
\hline $\begin{array}{l}\text { Cruz et al. } \\
\quad[28]\end{array}$ & 2019 & $\begin{array}{l}\text { Walking, walking upstairs, } \\
\text { walking downstairs, sitting, } \\
\text { standing, and laying }\end{array}$ & $\begin{array}{c}\text { Three CNN models } \\
\text { Fine-CNN } \\
\text { Medium-CNN } \\
\text { Coarse-CNN }\end{array}$ & $A=100 \%$ \\
\hline $\begin{array}{l}\text { Chen et al. } \\
\text { [29] }\end{array}$ & 2015 & $\begin{array}{l}\text { Walking, Jumping, Walking } \\
\text { quickly, step walking, } \\
\text { walking upstairs, walking } \\
\text { downstairs, running }\end{array}$ & $\begin{array}{l}\text { Modified CNN } \\
\text { model }\end{array}$ & $\mathrm{A}=93.8 \%$ \\
\hline $\begin{array}{l}\text { Mukherjee } \\
\text { et al. [30] }\end{array}$ & 2020 & $\begin{array}{l}\text { Walking, running, cooking, } \\
\text { performing office work. }\end{array}$ & $\begin{array}{c}\text { CNN-Net } \\
\text { Encoded-Net } \\
\text { EnsemConvNet with } \\
\text { AWFA }\end{array}$ & $\begin{array}{l}A=96.4 \% \\
A=96.2 \% \\
A=97.1 \%\end{array}$ \\
\hline $\begin{array}{l}\text { Almaslukh } \\
\text { et al. [31] }\end{array}$ & 2018 & $\begin{array}{l}\text { Walking, climbing upstairs, } \\
\text { climbing down stairs, } \\
\text { jumping, lying, standing, } \\
\text { sitting, running/jogging }\end{array}$ & Robust CNN Model & F-measure $=98 \%$ \\
\hline $\begin{array}{l}\text { Masum et } \\
\text { al. [32] }\end{array}$ & 2019 & $\begin{array}{l}\text { Standing still, sitting and } \\
\text { relaxing, lying down, } \\
\text { walking, climbing stairs, } \\
\text { waist bends forward, the } \\
\text { frontal elevation of arms, } \\
\text { knees bending, cycling, } \\
\text { jogging, running, jump front } \\
\text { and back. }\end{array}$ & $\begin{array}{c}\text { RF } \\
\text { NB } \\
\text { SVM } \\
\text { MLP } \\
\text { Deep CNN }\end{array}$ & $\begin{array}{c}A=89.2 \% \\
A=71.4 \% \\
78.46 \% \\
A=91.6 \% \\
A=98.4 \%\end{array}$ \\
\hline $\begin{array}{l}\text { Ihianle et } \\
\text { al. [33] }\end{array}$ & 2020 & $\begin{array}{c}12 \text { activities from } \\
\text { MHEALTH }+17 \text { activities } \\
\text { from WISDM }\end{array}$ & $\begin{array}{c}\text { CNN } \\
\text { Bi-LSTM } \\
\text { MCBiLSTM } \\
\end{array}$ & $\begin{array}{l}\mathrm{A}=88.4 \% \\
\mathrm{~A}=92.4 \% \\
\mathrm{~A}=97.9 \%\end{array}$ \\
\hline
\end{tabular}

\section{Conclusion and Future Work}

The survey presented a set of studies for the recognition of the HAR in terms of machine and deep learning methodologies. The studies were summarized in terms of data, features, and classification methods. The deep learning approach showed higher performance in accuracy and number of activities that are used and recognized 
than machine learning. Deep learning will improve the area of HAR by absorbing a huge amount of actions and activities for recognition. In summary, this survey aimed to consolidate the salient information on all aspects of HAR and to present this information for the benefit of the research community. The researchers will be all to get an answer to any possible questions on any stage of the HAR using this source. It is advised to apply more recent deep learning approaches in HAR. These methods can be 1D- convolutional neural networks, temporary convolutional networks (TCN), and many other. The former networks can have the ability to deal with 1D data and time series data. It is also recommended to increase the number of actions and activities to obtain a complete system that is capable of recognition every single action.

\section{References}

1. Lara, O. D., \& Labrador, M. A. (2012). A survey on human activity recognition using wearable sensors. IEEE communications surveys \& tutorials, 15(3), 1192-1209.

2. Ramasamy Ramamurthy, S., \& Roy, N. (2018). Recent trends in machine learning for human activity recognition-A survey. Wiley Interdisciplinary Reviews: Data Mining and Knowledge Discovery, 8(4), e1254.

3. Labrador, M. A., \& Yejas, O. D. L. (2013). Human activity recognition: Using wearable sensors and smartphones. CRC Press.

4. Akl, A., Taati, B., \& Mihailidis, A. (2015). Autonomous unobtrusive detection of mild cognitive impairment in older adults. IEEE transactions on biomedical engineering, 62(5), 1383-1394.

5. LeCun, Y., \& Bengio, Y. (1995). Convolutional networks for images, speech, and time series. The handbook of brain theory and neural networks, 3361(10), 1995.

6. Alam, M. A. U., Roy, N., Gangopadhyay, A., \& Galik, E. (2017). A smart segmentation technique towards improved infrequent non-speech gestural activity recognition model. Pervasive and Mobile Computing, 34, 25-45.

7. Khan, M. A., Ngo, H. H., Guo, W. S., Liu, Y., Nghiem, L. D., Hai, F. I., ... \& Wu, Y. (2016). Optimization of process parameters for production of volatile fatty acid, biohydrogen, and methane from anaerobic digestion. Bioresource technology, 219, 738-748.

8. Faridee, A. Z. M., Khan, M. A. A. H., Pathak, N., \& Roy, N. (2019, November). AugToAct: Scaling complex human activity recognition with few labels. In Proceedings of the 16th EAI International Conference on Mobile and Ubiquitous Systems: Computing, Networking, and Services (pp. 162-171).

9. Hussain, Z., Sheng, M., \& Zhang, W. E. (2019). Different approaches for human activity recognition: A survey. arXiv preprint arXiv:1906.05074.

10. Plötz, T., Hammerla, N. Y., \& Olivier, P. L. (2011, June). Feature learning for activity recognition in ubiquitous computing. In the Twenty-second international joint conference on artificial intelligence.

11. Bao, L., \& Intille, S. S. (2004, April). Activity recognition from user-annotated acceleration data. In International conference on pervasive computing (pp. 1-17). Springer, Berlin, Heidelberg.

12. Kwapisz, J. R., Weiss, G. M., \& Moore, S. A. (2011). Activity recognition using cell phone accelerometers. ACM SigKDD Explorations Newsletter, 12(2), 74-82.

13. Sharma, A., Lee, Y. D., \& Chung, W. Y. (2008, November). High accuracy human activity monitoring using neural network. In 2008 third international conference on convergence and hybrid information technology (Vol. 1, pp. 430-435). IEEE.

14. Khan, A. M. (2013). Recognizing physical activities using Wii remote. International Journal of Information and Education Technology, 3(1), 60.

15. Shoaib, M., Bosch, S., Incel, O. D., Scholten, H., \& Havinga, P. J. (2015). A survey of online activity recognition using mobile phones. Sensors, 15(1), 2059-2085.

16. Anguita, D., Ghio, A., Oneto, L., Parra, X., \& Reyes-Ortiz, J. L. (2012, December). Human activity recognition on smartphones using a multiclass hardware-friendly support vector machine. In International workshop on ambient assisted living (pp. 216-223). Springer, Berlin, Heidelberg. 
17. Tran, D. N., \& Phan, D. D. (2016, January). Human activities recognition in android smartphone using support vector machine. In 2016 7th international conference on intelligent systems, modeling, and simulation (isms) (pp. 64-68). IEEE.

18. Gusain, K., Gupta, A., \& Popli, B. (2018). Transition-aware human activity recognition using extreme gradient boosted decision trees. In Advanced Computing and Communication Technologies (pp. 41-49). Springer, Singapore.

19. Zdravevski, E., Lameski, P., Trajkovik, V., Kulakov, A., Chorbev, I., Goleva, R., \& Garcia, N. (2017). Improving activity recognition accuracy in ambient-assisted living systems by automated feature engineering. Ieee Access, 5, 5262-5280.

20. Bao, L., \& Intille, S. S. (2004, April). Activity recognition from user-annotated acceleration data. In International conference on pervasive computing (pp. 1-17). Springer, Berlin, Heidelberg.

21. Shoaib, M., Bosch, S., Incel, O. D., Scholten, H., \& Havinga, P. J. (2014). Fusion of smartphone motion sensors for physical activity recognition. Sensors, 14(6), 10146-10176.

22. Khan, A. M., Lee, Y. K., Lee, S. Y., \& Kim, T. S. (2010). A triaxle accelerometer-based physical-activity recognition via augmented-signal features and a hierarchical recognizer. IEEE transactions on information technology in biomedicine, 14(5), 1166-1172.

23. Xu, C., Chai, D., He, J., Zhang, X., \& Duan, S. (2019). InnoHAR: A deep neural network for complex human activity recognition. Ieee Access, 7, 9893-9902.

24. Hossain, H. S., Khan, M. A. A. H., \& Roy, N. (2017). Active learning-enabled activity recognition. Pervasive and Mobile Computing, 38, 312-330.

25. Gjoreski, M., Janko, V., Slapničar, G., Mlakar, M., Reščič, N., Bizjak, J. \& Gams, M. (2020). Classical and deep learning methods for recognizing human activities and modes of transportation with smartphone sensors. Information Fusion, 62, 47-62.

26. Zebin, T., Scully, P. J., \& Ozanyan, K. B. (2016, October). Human activity recognition with inertial sensors using a deep learning approach. In 2016 IEEE SENSORS (pp. 1-3). IEEE.

27. Shi, X., Li, Y., Zhou, F., \& Liu, L. (2018, August). Human activity recognition based on deep learning method. In 2018 International Conference on Radar (RADAR) (pp. 1-5). IEEE.

28. Avilés-Cruz, C., Ferreyra-Ramírez, A., Zúñiga-López, A., \& Villegas-Cortéz, J. (2019). Coarse-fine convolutional deep-learning strategy for human activity recognition. Sensors, 19(7), 1556.

29. Chen, Y., \& Xue, Y. (2015, October). A deep learning approach to human activity recognition based on a single accelerometer. In 2015 IEEE international conference on systems, man, and cybernetics (pp. 14881492). IEEE.

30. Mukherjee, D., Mondal, R., Singh, P. K., Sarkar, R., \& Bhattacharjee, D. (2020). EnsemConvNet: a deep learning approach for human activity recognition using smartphone sensors for healthcare applications. Multimedia Tools and Applications, 79(41), 31663-31690.

31. Almaslukh, B., Artoli, A. M., \& Al-Muhtadi, J. (2018). A robust deep learning approach for positionindependent smartphone-based human activity recognition. Sensors, 18(11), 3726.

32. Masum, A. K. M., Hossain, M. E., Humayra, A., Islam, S., Barua, A., \& Alam, G. R. (2019, April). A statistical and deep learning approach for human activity recognition. In 2019 3rd International Conference on Trends in Electronics and Informatics (ICOEI) (pp. 1332-1337). IEEE.

33. Ihianle, I. K., Nwajana, A. O., Ebenuwa, S. H., Otuka, R. I., Owa, K., \& Orisatoki, M. O. (2020). A deep learning approach for human activity recognition from multimodal sensing devices. IEEE Access, 8, 179028-179038. 